\title{
Effect of Transcranial Direct Current Stimulation on Postural Stability and Lower Extremity Strength in Hemiplegic Stroke Patients
}

\author{
Min Kyun Sohn, MD, Sung Ju Jee, MD, Yeong Wook Kim, MD
}

Department of Rehabilitation Medicine, Chungnam National University School of Medicine, Daejeon, Korea

\begin{abstract}
Objective To evaluate the effect of anodal transcranial direct current stimulation (tDCS) over the lesioned leg motor cortex, which can enhance the strength and coordination of the contralateral lower extremity and furthermore, enhance the postural stability of the hemiplegic subject.

Methods Anodal or sham stimulation on the lesioned cortex of a lower extremity was delivered to 11 ambulatory hemiplegic patients. The stimulation intensity was $2 \mathrm{~mA}$. All subjects took part in two 10-minute tDCS sessions consisting of anodal stimulation and sham stimulation. The interval period between real and sham stimulation was 48 hours. The order was counter-balanced among the subjects. Before and after each stimulation session, static postural stability was evaluated with eyes opened and closed. Also, the isometric strength of the hemiplegic side of the treated knee was measured before and after each stimulation session. Repeated measure ANOVA was used to determine the statistical significance of improvements in postural stability and strength.

Results There was significant improvement for overall stability index with eyes opened and closed after anodal tDCS $(p<0.05)$. Isometric strength of the lesioned quadriceps tended to increase after anodal tDCS ( $<<0.05)$. Postural stability and quadriceps strength were not changed after sham stimulation.

Conclusion Anodal tDCS has potential value in hemiplegic stroke patients to improve balance and strengthen the affected lower extremity.
\end{abstract}

Keywords tDCS, Postural balance, Stroke, Strength

Received May 16, 2013; Accepted August 13, 2013

Corresponding author: Yeong Wook Kim

Department of Rehabilitation Medicine, Chungnam National University Hospital, 282 Munhwa-ro, Jung-gu, Daejeon 301-721, Korea

Tel: +82-42-338-2460, Fax: +82-42-338-2461, E-mail: kyu0922@cnuh.co.kr

(c) This is an open-access article distributed under the terms of the Creative Commons Attribution Non-Commercial License (http://creativecommons. org/licenses/by-nc/3.0) which permits unrestricted noncommercial use, distribution, and reproduction in any medium, provided the original work is properly cited.

Copyright $\odot 2013$ by Korean Academy of Rehabilitation Medicine

\section{INTRODUCTION}

Stroke is a leading global cause of disability [1]. Neurological disorders like stroke can impair body functions most often movement [2]. Many stroke patients experience difficulties carrying out activities of daily living. They undergo rehabilitative therapies to improve their motor function. However, the impairment in movement is not fully recovered in many cases, and stroke patients live with the stroke-related disabilities [1]. With the aim for improving motor function after stroke and minimiz- 
ing disabilities, various rehabilitative therapies have been developed [3].

The balance impairment in patients with hemiplegia after stroke is usually caused by loss of muscle strength and coordination, spasticity, neurological disorders or degenerative disorder [4]. The risk for falls increases, and activities of daily living functioning is affected as a result. Falling accidents can cause serious results, such as fracture and traumatic brain damage [5]. Pelvic fracture is the most common injury caused by falls, and it take a great amount of time to recover when it occurs in elderly people [6].

Transcranial direct current stimulation (tDCS) is a noninvasive brain stimulation approach that is increasingly generated interest [7] as a means increasing or decreasing cortical excitability, depending on the delivery of anodal or cathodal stimulation to the cerebral cortex.

Many studies have focused on the effects of tDCS of the cerebral cortex on upper limb function. Nitsche and Paulus [8] reported the temporary increase and decrease of the cortical excitability in response to tDCS anodal or cathodal stimulation, respectively, delivered to the upper limb area of the primary motor cortex. Hummel et al. [9] reported improved activities of daily living including hand movements in stroke survivors when the upper limb area of the motor cortex was stimulated by tDCS in addition to palliative rehabilitative intervention. Kim et al. [10] reported tDCS was effective in improving the function of the hemiplegic side of the affected upper limb and that this improved function continued after stimulation.

Stroke can also lead to gait disability by affecting lower limb movement and function as it does to the upper limb. Studies that investigated effects of tDCS on lower limb function have been more recent. In 2007, Jeffery et al. [11] reported an increase in the amplitude of motor evoked potential (MEP) in the tibialis anterior muscle after tDCS on the leg area of primary motor cortex. In 2009, Tanaka et al. [12] claimed that the muscle strength of toes on the contralateral side improved temporarily as anodal tDCS was administered to the leg area of the primary motor cortex in healthy adults. In 2011, the same authors reported a statistically significant improvement in knee extensor strength in the hemiplegic side when anodal tDCS was delivered on the ipsilesional leg area of the primary motor cortex via tDCS in chronic stroke patients [13].
Studies related to tDCS have concentrated on upper limb function rather than lower limb. Still, no study has investigated effects of tDCS on balance. We investigated effects of tDCS on the leg motor function measured by balance performance, posture control function and knee extensor strength by applying anodal stimulation on the leg area of the primary motor cortex in subacute stroke patients with hemiplegia.

\section{MATERIALS AND METHODS}

\section{Patients}

Eleven stroke patients with hemiplegia were selected from patients undergoing rehabilitation following acute treatment. The average age was $58.45 \pm 14.55$ years and the average prevalence period was $63.00 \pm 17.27$ days. Four patients suffered from cerebral infarction and seven patients had cerebral hemorrhage. All patients were receiving rehabilitative care with an average length of stay was less than six months after the onset. Cerebral hemorrhage and cerebral infarction were diagnosed using computed tomography and magnetic resonance imaging

Table 1. Characteristics of the 11 subjects

\begin{tabular}{|cc|}
\hline Characteristic & Value \\
\hline Sex & $9(81.8)$ \\
\hline Male & $2(18.2)$ \\
\hline Female & $58.45 \pm 14.55$ \\
\hline Age $(\mathrm{yr})$ & $170.02 \pm 9.48$ \\
\hline Height $(\mathrm{cm})$ & $67.39 \pm 17.27$ \\
\hline Weight $(\mathrm{kg})$ & \\
\hline Stroke type & \\
\hline CI & $4(36.4)$ \\
\hline Striatocapsular & $6(54.6)$ \\
\hline S-ICH & $1(9.0)$ \\
\hline Basal ganglia & \\
\hline Thalamus & $6(54.5)$ \\
\hline Hemiplegic side & $5(45.5)$ \\
\hline Right & $63.00 \pm 17.27$ \\
\hline Left & \\
\hline Onset duration (day) & \\
\hline
\end{tabular}

Values are presented as mean \pm standard deviation or number (\%).

S-ICH, spontaneous intracerebral hemorrhage; CI, cerebral infarction. 
(MRI). All patients had knee extensor strength gauged as fair or better in the hemiplegic side with the ability to maintain balanced standing and sitting positions. At the same time, their Korean Mini-Mental State Examination score was 24 or higher, indicative of cognitive ability sufficient to understand the experiment. Any patients with previous stroke or previous epilepsy/seizure, family history of epilepsy/seizure, any metal substance inserted into the cranial cavity, permanent pacemaker, previous and present other neurological disorders, stroke lesion in cerebellum and contracture of the lower limb on the affected side of the lesion were excluded [10] (Table 1).

\section{Study methods}

Patients completed the experiment in which real and sham stimulation were applied to the leg area of the primary motor cortex directly at a 48-hour interval to minimize the influence of the first outcome on the other [1416]. The two stimulation experiments were performed in random order for each patient. Knee extensor strength and balance performance were measured four times before and after real and sham stimulation (Fig. 1).
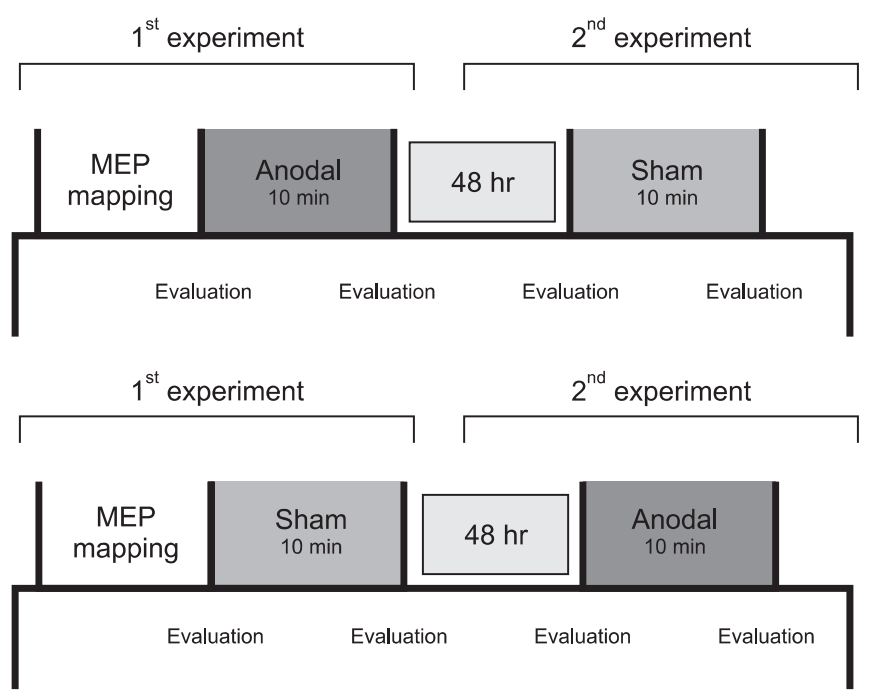

Fig. 1. Patients completed the experiment in which real and sham stimulation were applied to the leg area of the primary motor cortex. The two stimulation experiments were performed in random order for each patient. Knee extensor strength and balance performance were measured three times before and after real and sham stimulation. MEP, motor evoked potential.

\section{tDCS}

Phoresor II Auto model PM 850 (IOMED, Salt Lake City, UT, USA) was used. The square sponge electrode placed on the head was $5 \mathrm{~cm} \times 5 \mathrm{~cm}$. Anodal stimulation was delivered at a level of $2 \mathrm{~mA}$ for 10 minutes. Once tDCS was activated, the intensity of stimulation was set to increase slowly for the first 15 seconds and decreased gradually for the last 15 seconds. This intensity level is appropriate to elicit excitability of the leg primary motor cortex without causing complications [11]. Sham stimulation was also delivered at a level of $2 \mathrm{~mA}$ for the first 10 seconds and was turned off after 10 seconds without the knowledge of the patients, and was activated again after 10 minutes for only 10 seconds. Patients were unlikely to be aware of any difference between real and sham stimulation [11]. Electrodes were placed on the hot spot-the stimulation site where quadriceps femoris muscle MEPs were easily obtained in the primary motor cortex of the affected side. In case the hot spot could not be defined on the motor cortex of the affected side, it could be acquired by the same position of hot spot of the unaffected side to apply the anodal electrode [10]. For three patients whose hot spot did not be defined on the motor cortex of the affected side, the cathodal electrode was placed on the supraorbital area on the unaffected side.

\section{Balance assessment}

Balance performance was assessed using the Balance System SD (Biodex Medical Systems, Shirley, NY, USA). This assessment tool is equipped with a movable balance platform and is designed to provide $20^{\circ}$ of platform tilt in a $360^{\circ}$ range of motion. The static platform system allows the assessment of static stability during standing on one foot or both feet. In this study, static balance was assessed in standing position on a static platform with both feet. Patients stood on both feet comfortably with knees fixed $\left(10^{\circ}-15^{\circ}\right)$ and while staring straight ahead with arms crossed on the chest. Feet position remained stable during the test [17]. Three tests were carried out for $20 \mathrm{sec}-$ onds each, allowing a break of 10 seconds between tests. Each balance stability results represented an average of three measurements. Static stability was measured with eyes open and closed. Overall stability index, anterior/ posterior stability index and medial/lateral stability index were measured. To minimize the training effect resulted from continued testing, training was provided three times 
before testing [17].

\section{Isometric strength of knee extensor muscle}

Knee extensor strength was measured using Biodex System 4 Pro (Biodex Medical Systems). Patients were told to sit upright on a seat of the muscle tester and their body was fixed to the chair with a three-point belt. With the axis of the knee on the affected side being aligned with the axis of the tester, the two leg straps were tightened in distal femur and ankle to restrict body movement. Patients were told about tests and methods for 10 minutes and practiced before the test began. Testing time and isometric muscle activation level were electronically monitored, making biofeedback display possible. To measure the isometric strength of knee extensor muscle, patients were encouraged to maximize force production for $5 \mathrm{sec}$ onds with the knee joint fixed at an angle of $60^{\circ}$ [18]. Each test took 5 seconds, followed by a 5 -second break. Three tests were carried out. The result was based on an average of three tests.

\section{Statistical analysis}

Statistical analyses were performed using a Korean version of SPSS 18.0 (SPSS Inc., Chicago, IL, USA). Mean value and standard deviation were computed for isometric strength of the knee extensor muscle and maintenance of balance measured before and after real stimulation and sham stimulation. Changes (\%) of indices after real and sham stimulation were computed. Wilcoxon signed rank test was used to compare the results between real and sham stimulation. Spearman correlation was also performed to test the association between strength of knee extensor muscle and stability index. A p-value $<0.05$ was considered statistically significant.

\section{RESULTS}

\section{Isometric knee extensor power}

Change of isometric peak torque for knee extensor from baseline was $20.62 \% \pm 17.70 \%$ after real stimulation and $2.43 \% \pm 6.08 \%$ after sham stimulation $(\mathrm{p}<0.05)$ (Fig. $2 \mathrm{~A}$ ). Change of average peak torque for knee extensor from baseline was $16.91 \% \pm 12.77 \%$ after real stimulation and $4.71 \% \pm 8.82 \%$ after sham stimulation $(\mathrm{p}<0.05)$ (Fig. $2 \mathrm{~B}$ ).

\section{Balance assessment}

After real stimulation, overall, anterior/posterior, and medial/lateral stability index with eyes open change was $-59.86 \% \pm 40.89 \%,-71.44 \% \pm 70.06 \%$, and $-58.87 \% \pm 119.27 \%$, respectively. After sham stimulation, the respective change was $-6.45 \% \pm 17.99 \%,-7.73 \% \pm 32.69 \%$, and $2.86 \% \pm 20.93 \%(\mathrm{p}<0.05)$ (Fig. $3 \mathrm{~A})$. With eyes closed, the respective change was $-70.15 \% \pm 68.49 \%,-53.09 \% \pm 65.12 \%$, and $-112.66 \% \pm 146.82 \%$, respectively. After sham stimulation the respective change was $-5.21 \% \pm 15.17 \%$, $-7.10 \% \pm 13.59 \%$, and $5.64 \% \pm 22.09 \%(\mathrm{p}<0.05)$ (Fig. 3B).

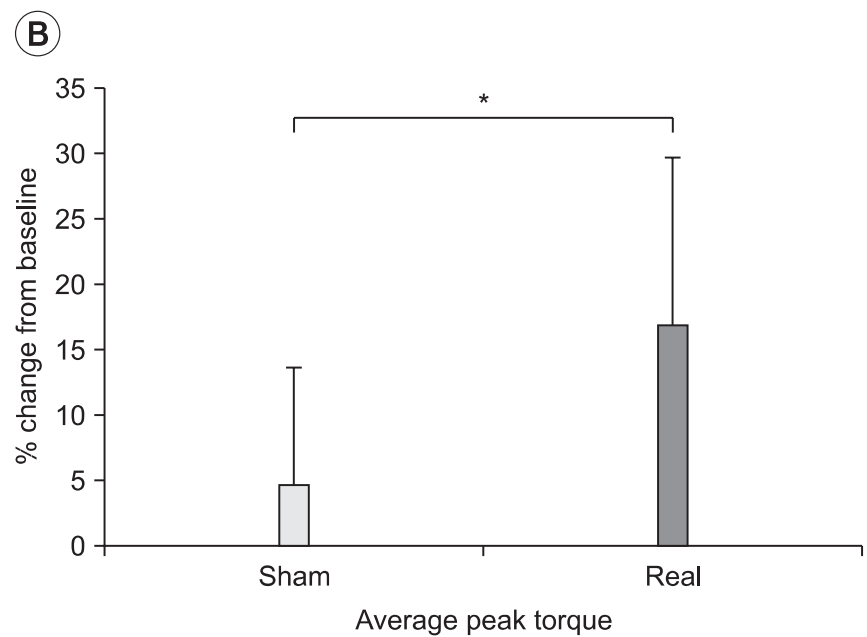

Fig. 2. Effects of transcranial direct current stimulation (tDCS) on peak torque and average peak torque of the isometric knee extensor. The isometric knee extensor increased after real anodal tDCS over primary motor cortex compared to sham stimulation. (A) Peak torque, (B) average peak torque. ${ }^{*} \mathrm{p}<0.05$. 
(A)

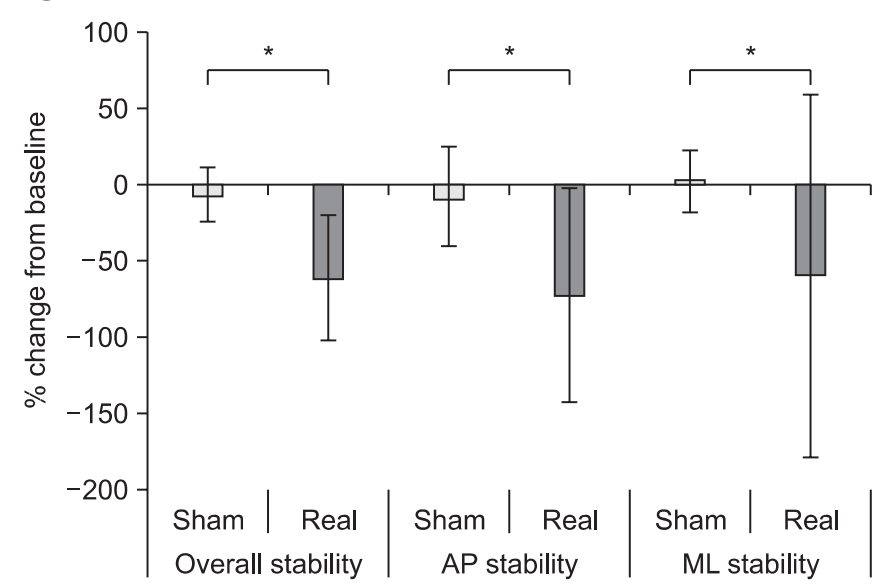

(B)

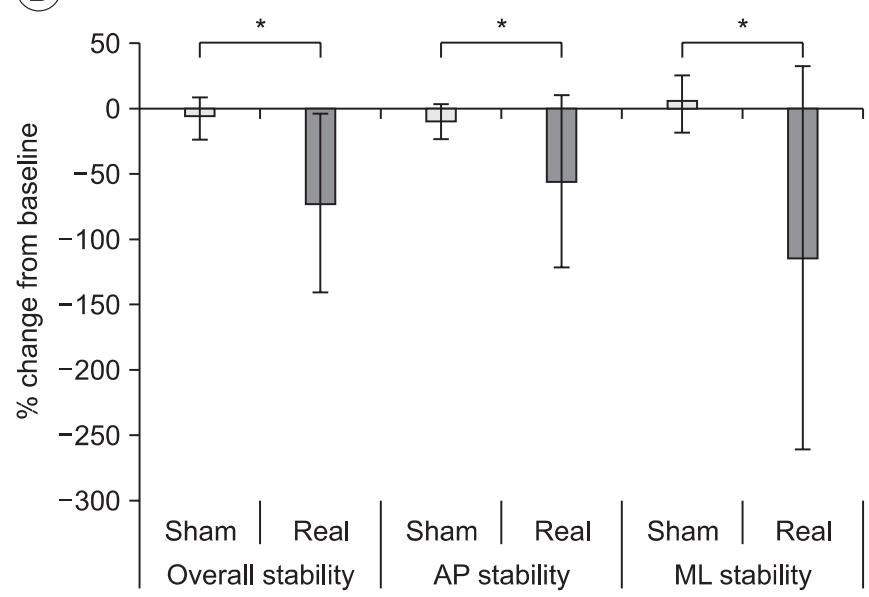

Fig. 3. Effects of transcranial direct current stimulation (tDCS) on the static stability. Static stability index with eyes open (A) and closed (B) decreased after real anodal tDCS over primary motor cortex compared to sham stimulation. $\mathrm{AP}$, anterior/posterior; ML, medial/lateral. ${ }^{*} \mathrm{p}<0.05$.

Table 2. Spearman rank correlation coefficient between peak torque of knee extensor and stability index at baseline with eyes open

\begin{tabular}{lcc}
\hline & $\begin{array}{c}\text { Average peak } \\
\text { torque }\end{array}$ & Peak torque \\
\hline Overall stability & -0.456 & -0.416 \\
AP stability & -0.304 & -0.260 \\
ML stability & -0.320 & -0.282 \\
\hline
\end{tabular}

AP, anterior/posterior; ML, medial/lateral.

Correlation between knee extensor strength and balance performance

Pre-tDCS average peak torque and knee extensor peak torque and balance performance with eyes open was examined. No statistically significant correlation was found (Table 2).

\section{DISCUSSION}

This study investigated the effects of anodal tDCS administered to the leg area of the primary motor cortex on the lower limb muscle strength and balance performance in stroke patients with hemiplegia. The study was designed with the assumption that impaired leg strength resulting from the hemiplegia would affect balance performance. This means that improved muscle strength of the leg by anodal tDCS would increase balance performance significantly. Tomas-Carus et al. [19] suggested a cor- relation between knee extensor strength and balance in patients with fibromyalgia. Tanaka et al. [12] claimed that improvement in contralateral side of lower limb muscle strength was elicited by anodal tDCS. Effects of anodal tDCS has been proven in stroke patients with hemiplegia as their lower limb movement control improved after anodal tDCS to the leg primary motor cortex on the affected side [16].

This study also found a significant increase in knee extensor peak torque after anodal tDCS on the leg area of the affected primary motor cortex, when compared with baseline measurement. Also, in the overall stability index, in which an increased score means a poor balance performance, also significantly decreased after the anodal tDCS. Thus, the results of this study support the hypothesis that tDCS of the leg area of the primary motor cortex on the affected side boosts lower limb muscle strength, which also leads to benefits in balance performance.

The results are consistent with those of earlier studies in anodal tDCS delivered to the hand or leg area of the primary motor cortex. Hummel et al. [9] and Fregni et al. [20] reported improved upper limb function after administering tDCS to the M1 area of the cerebral cortex on the affected area for 20 minutes in stroke patients. Although the recovery of walking ability is the most important goal for stroke patients [21], studies investigating noninvasive brain stimulations as a supplementary approach in improving lower limb function have only recently been carried out. Tanaka et al. [13] reported increased knee ex- 
tensor strength after anodal tDCS on the lower leg area of the primary motor cortex on the affected area in chronic stroke patients with hemiplegia. But, the effects of sham stimulation were not significant. In this study, effects of tDCS on balance performance were also found.

Previous studies have used functional MRI (fMRI) to assess changes resulting from hand and finger movement. In one study, anodal tDCS increased activation of the ipsilateral supplementary motor area and lowered the extent of activation of both anterior cingulated gyri, right middle and superior temporal gyri, middle and superior frontal gyri, and primary and secondary somatosensory cortices [22]. The authors reported that anodal tDCS modulated the activity of the leg motor cortex in healthy subjects, which supports the present results.

In a previous study, $\mathrm{tDCS}$ used to facilitating upper limb function was administered at $1 \mathrm{~mA}$, lower than the $2 \mathrm{~mA}$ used in this study. Jeffery et al. [11] found in a preliminary study that anodal stimulation delivered at the intensity of $1 \mathrm{~mA}$ failed to affect lower limb MEPs. This finding was likely due to the fact that the leg area of primary motor cortex is located further inside the motor cortex than the arm area. Any anodal stimulation administered at a level of $2 \mathrm{~mA}$ improves the amplitude of leg MEPs, which is similar to a $40 \%$ increase in the improvement achieved in amplitude of hand MEPs at a level of $1 \mathrm{~mA}$. This study also administered anodal tDCS at an intensity of $2 \mathrm{~mA}$ to accelerate the stimulation to the leg area of the primary motor cortex.

The correlation between pre-tDCS knee extensor peak torque and balance performance was not statistically significant. Factors that affect balance performance are strength, cerebellar function, visual acuity, proprioception, inner ear function, foot position on the ground, leg length discrepancy, medication, age, heartbeat, respiration, and auditory function. Further studies should include evaluations for all factors affecting balance performance, whether these factors would be affected by tDCS or not.

This study has some limitations. First, the number of subjects was too small to ensure statistical verification. Second, a double-blind design was not used for experiments. Third, the stimulation was intended for the primary motor cortex. But, given the nature of tDCS, the actual stimulation site extended beyond the primary motor cortex. It is therefore hard to estimate whether obtained improvement was elicited solely by a change in the primary motor cortex or whether other areas of the cerebral cortex also contributed to the improvement. Further study needs to include neuroimaging analysis. Fourth, despite improvement in muscle strength and balance performance in the hemiplegic side, the association between muscle strength and balance performance could not be clearly identified because of the lack of electrophysiological index, such as MEP, on the affected side of the lesion. Fifth, we included patients with either cerebral hemorrhage or cerebral infarction. So, we could not explain the different effects of tDCS on balance between cerebral hemorrhagic lesion and cerebral infarction lesion.

Despite limitations, this study is distinguished from previous studies by proving effects of tDCS on balance performance, which is a crucial element in walking ability and preventing falls. Given that walking ability is the most desired treatment outcome for many patients, previous studies examined excitability of the cerebral cortex, lower limb movement and muscle strength as beneficial areas of tDCS. The use of tDCS as a supplementary application in addition to rehabilitation in stroke patients is expected to optimize treatment outcomes.

In conclusion, this study observed the improvement in isometric knee extensor strength and balance performance after anodal tDCS of the primary motor cortex on the affected side of the lesion in subacute stroke patients with hemiplegia who can walk. Anodal tDCS administered to the primary motor cortex may be an effective supplementary choice for improving lower limb muscle strength and balance performance in stroke patients seeking rehabilitation.

\section{CONFLICT OF INTEREST}

No potential conflict of interest relevant to this article was reported.

\section{REFERENCES}

1. Kolominsky-Rabas PL, Weber M, Gefeller O, Neundoerfer B, Heuschmann PU. Epidemiology of ischemic stroke subtypes according to TOAST criteria: incidence, recurrence, and long-term survival in ischemic stroke subtypes: a population-based study. Stroke 
2001;32:2735-40.

2. Hankey GJ, Jamrozik K, Broadhurst RJ, Forbes S, Anderson CS. Long-term disability after first-ever stroke and related prognostic factors in the Perth Community Stroke Study, 1989-1990. Stroke 2002;33:1034-40.

3. Johansson BB. Current trends in stroke rehabilitation: a review with focus on brain plasticity. Acta Neurol Scand 2011;123:147-59.

4. Geurts AC, de Haart M, van Nes IJ, Duysens J. A review of standing balance recovery from stroke. Gait Posture 2005;22:267-81.

5. Rubenstein LZ, Josephson KR. The epidemiology of falls and syncope. Clin Geriatr Med 2002;18:141-58.

6. Tinetti ME, Williams CS. The effect of falls and fall injuries on functioning in community-dwelling older persons. J Gerontol A Biol Sci Med Sci 1998;53:M112-9.

7. Nitsche MA, Boggio PS, Fregni F, Pascual-Leone A. Treatment of depression with transcranial direct current stimulation (tDCS): a review. Exp Neurol 2009; 219:14-9.

8. Nitsche MA, Paulus W. Excitability changes induced in the human motor cortex by weak transcranial direct current stimulation. J Physiol 2000;527 Pt 3:633-9.

9. Hummel F, Celnik P, Giraux P, Floel A, Wu WH, Gerloff $\mathrm{C}$, et al. Effects of non-invasive cortical stimulation on skilled motor function in chronic stroke. Brain 2005; 128(Pt 3):490-9.

10. Kim DY, Park CI, Jung KJ, Ohn SH, Park KD, Park JB, et al. Improvement of chronic post-stroke hemiparetic upper limb function after 2 week trascranial direct current stimulation. J Korean Acad Rehabil Med 2009;33:5-11.

11. Jeffery DT, Norton JA, Roy FD, Gorassini MA. Effects of transcranial direct current stimulation on the excitability of the leg motor cortex. Exp Brain Res 2007;182:281-7.

12. Tanaka S, Hanakawa T, Honda M, Watanabe K. Enhancement of pinch force in the lower leg by anodal transcranial direct current stimulation. Exp Brain Res 2009;196:459-65.

13. Tanaka S, Takeda K, Otaka Y, Kita K, Osu R, Honda
$M$, et al. Single session of transcranial direct current stimulation transiently increases knee extensor force in patients with hemiparetic stroke. Neurorehabil Neural Repair 2011;25:565-9.

14. Fertonani A, Rosini S, Cotelli M, Rossini PM, Miniussi C. Naming facilitation induced by transcranial direct current stimulation. Behav Brain Res 2010;208:311-8.

15. Kang EK, Paik NJ. Effect of a tDCS electrode montage on implicit motor sequence learning in healthy subjects. Exp Transl Stroke Med 2011;3:4.

16. Madhavan S, Weber KA 2nd, Stinear JW. Non-invasive brain stimulation enhances fine motor control of the hemiparetic ankle: implications for rehabilitation. Exp Brain Res 2011;209:9-17.

17. Aydog E, Depedibi R, Bal A, Eksioglu E, Unlu E, Cakci A. Dynamic postural balance in ankylosing spondylitis patients. Rheumatology (Oxford) 2006;45:445-8.

18. Lee H, Noh GB, Lee YH, Seong NJ, Lee HC. Effect of concentric isokinetic knee strength training on gait, balance and quality of life in chronic stroke patients. J Korean Acad Rehabil Med 2007;31:649-54.

19. Tomas-Carus P, Gusi N, Hakkinen A, Hakkinen K, Raimundo A, Ortega-Alonso A. Improvements of muscle strength predicted benefits in HRQOL and postural balance in women with fibromyalgia: an 8-month randomized controlled trial. Rheumatology (Oxford) 2009;48:1147-51.

20. Fregni F, Boggio PS, Mansur CG, Wagner T, Ferreira MJ, Lima MC, et al. Transcranial direct current stimulation of the unaffected hemisphere in stroke patients. Neuroreport 2005;16:1551-5.

21. Jorgensen HS, Nakayama H, Raaschou HO, Olsen TS. Recovery of walking function in stroke patients: the Copenhagen Stroke Study. Arch Phys Med Rehabil 1995;76:27-32.

22. Kim CR, Kim DY, Kim LS, Chun MH, Kim SJ, Park $\mathrm{CH}$. Modulation of cortical activity after anodal transcranial direct current stimulation of the lower limb motor cortex: a functional MRI study. Brain Stimul 2012;5:462-7. 